\title{
Chemotherapy-Induced Nausea and Vomiting in Gastrointestinal Cancer Patients: Do We Need to Revisit Guidelines?
}

\author{
Akhil Kapoor ${ }^{1}$ Ashutosh Jain ${ }^{2} \quad$ Abhishek Sharma ${ }^{2}$ Minit Shah² Shravan Chinthala ${ }^{2}$ \\ Ravindra Nandhana ${ }^{2}$ Prabhat Bhargava ${ }^{2}$ Anant Ramaswamy ${ }^{2}$ Sujay Srinivas ${ }^{2}$ Vikas Ostwal $^{2}$ \\ 1Department of Medical Oncology, Homi Bhabha Cancer Hospital, \\ Homi Bhabha National Institute (HBNI), Varanasi, Uttar Pradesh, \\ India \\ 2Department of Medical Oncology, Tata Memorial Hospital, Homi \\ Bhabha National Institute (HBNI), Dr. E Borges Road, Parel, \\ Mumbai, Maharashtra, India \\ Address for correspondence Vikas Ostwal, DM, Department of \\ Medical Oncology, Tata Memorial Hospital, Homi Bhabha National \\ Institute (HBNI), Dr. E Borges Road, Parel, Mumbai 400012, \\ Maharashtra, India (e-mail: dr.vikas.ostwal@gmail.com).
}

\section{South Asian J Cancer 2021;9:245-249.}

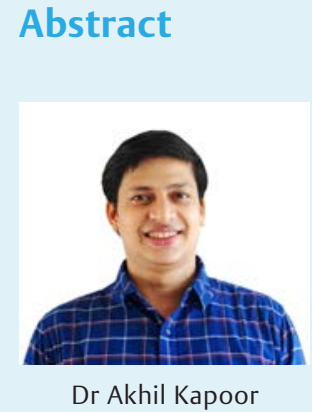

Keywords

- CINV

- moderately emetogenic chemotherapy

- MEC

- oxaliplatin

- complete response

\section{Introduction}

Chemotherapy-induced nausea and vomiting (CINV) is one of the most bothersome adverse effects of cancer chemotherapy and is implicated in reducing the quality of life and altering compliance to treatment. ${ }^{1}$

\section{DOI https://doi.org/10.1055/s-0041-1729493 ISSN 2278-330X}

How to cite this article: Kapoor A, Jain A, Sharma A, et al. Chemotherapy-Induced Nausea and Vom-iting in Gastrointestinal Cancer (GI) Patients: do we need to Revisit Guidelines? South Asian J Cancer 2021;9(4):245-249.
The patients may also develop complications secondary due to CINV, including anorexia, dehydration, and hyponatremia, besides entailing logistic issues. ${ }^{2}$ Various guidelines are available with regard to antiemetic prophylaxis (AEP) with the Multinational Association of Supportive Care in Cancer/European Society for Medical Oncology

(c) 2021. MedIntel Services Pvt Ltd.

This is an open access article published by Thieme under the terms of the Creative Commons Attribution-NonDerivative-NonCommercial-License, permitting copying and reproduction so long as the original work is given appropriate credit. Contents may not be used for commercial purposes, or adapted, remixed, transformed or built upon. (https://creativecommons.org/licenses/by-nc-nd/4.0/).

Thieme Medical and Scientific Publishers Private Ltd A-12, Second Floor, Sector -2, NOIDA -201301, India 
(MASCC/ESMO) and American Society of Clinical Oncology (ASCO) are the most commonly followed and cited., These classify the potential of CINV as per chemotherapy drug/regimen into high, moderate, and low emetogenic chemotherapeutic (HEC, MEC, and LEC, respectively) regimens. CINV comprises of early and delayed onset, both with their own purported mechanisms. ${ }^{5}$

Gastrointestinal (GI) cancer chemotherapy entails significant use of oxaliplatin and irinotecan in a majority of patients, thereby classifying most regimens as MEC. However, there is limited data assessing CINV in patients with GI cancers. This study focused on GI cancer patients with the primary objective to assess the complete response (CR) rates with existing antiemetic prophylaxis.

\section{Patients and Methods}

\section{Eligibility Criteria}

Eighteen years or older chemo-naïve patients with GI malignancy and the Eastern Cooperative Oncology Group (ECOG) performance status of 0 to 2 were eligible for enrolment in the study. Patients with symptomatic brain metastases and psychiatric/cognitive dysfunction, possibly interfering with the compliance to antiemetic therapy or with clinical evidence of current or impending bowel obstruction, were excluded from the study. The patients who were unable to maintain the diary were also excluded.

\section{Study Design and Oversight}

This was an observational study in which patients receiving chemotherapy for GI cancers (stomach, pancreas, gallbladder, and colorectal cancers) at Tata Memorial Hospital, Mumbai, between April 2019 and March 2020 were included. Patients were counseled to use a diary to record the number of vomiting episodes, intensity, and duration of nausea in the 5 days of postchemotherapy as part of standard of care in our unit. The primary endpoint of the study was an assessment of CR rates, defined as an absence of vomiting, significant nausea, or the need for rescue medications. The study was conducted in accordance with GCP (good clinical practice) ICH (International Council for Harmonization) and the Declaration of Helsinki principles. Written informed consent was obtained from all patients. The data were collected by trained medical coordinators. The authors vouch for the accuracy and completeness of the data and analysis and for adherence to the study protocol.

\section{Treatment Regimen}

The doses of the antiemetic used in the study were as per the standard recommendations and available studies published from India. ${ }^{4-7}$

All participants receiving HEC regimen received a $5 \mathrm{HT} 3$ (5-hydroxytryptamine) receptor antagonist (0.25-mg palonosetron intravenously or 1-mg granisetron intravenously, with the specific agent chosen by the primary clinician) on day 1 of chemotherapy, dexamethasone (8-12 mg intravenously on day 1 and 8-mg orally on days 2, 3, and 4), an NK1 (neurokinnin 1) receptor antagonist (125-mg aprepitant orally on day 1 and $80 \mathrm{mg}$ on days 2 and 3, or 150 mg fosaprepitant intravenously on day 1 ), and olanzapine (10 mg per day orally from day 1-4) in the selected cohort.

All participants receiving an MEC regimen received a two-drug combination of a 5-HT3 receptor antagonist and dexamethasone (8-12 mg intravenously on day 1). The patients receiving carboplatin were also given an NK1 receptor antagonist, and patients with LEC regimen received either a single dose of a 5 -HT3 receptor antagonist or a single 8-mg dose of dexamethasone before chemotherapy treatment. The patients receiving minimal emetogenic chemotherapy did not receive any premedication.

It should be noted that carboplatin-based regimens and FOLFIRINOX (modified 5-FU/LV-irinotecan-oxaliplatin) were classified as an MEC regimen.

\section{Statistical Analysis}

A descriptive analysis was performed on all of the variables (demographic variables and characteristics of the patients, the disease, and the chemotherapy received). For the primary objective, the incidence of CINV was evaluated during the 5 days following administration. CINV within the first 24 hours following administration of the chemotherapy (acute phase) and during the 4 subsequent days (delayed phase) were recorded and expressed as percentages. Logistic regression analysis was performed to identify significant factors affecting CINV. A p-value of less than 0.05 was considered statistically significant. SPSS version 26 (Armonk, New York, United States) was used for all statistical calculations.

\section{Results}

\section{Patients and Demographics}

A total of 1,062 cycles of chemotherapy from April 2019 to March 2020 was administered to these patients. Briefly, the median age of the patients was 53 years (range: $18-77$ years) with $57.3 \%$ being males. Biliary tract cancer (31.7\%) was the most common diagnosis followed by colorectal (29.2\%) and gastric cancer (19.7\%). Out of the 1,062 cycles delivered, 211 (9.8\%) were HECs, 613 (57.7\%) were MECs, 219 (20.6\%) were LECs, while 19 (1.8\%) were minimal emetogenic.

\section{Control of Nausea and Vomiting}

The rates of CR of CINV during acute and delayed periods are shown in - Table 1 . CR was not achieved in $23.2 \%$ of all cycles. CR was not achieved in $28.2 \%$ of the MEC regimen cycles, followed by $16.9 \%$ of LEC regimen cycles, and $16.6 \%$ of HEC regimen cycles. The loss of CR was similar with cycle 1 versus subsequent cycles (- Table 2).

For MEC regimens, the failure to achieve CR rates was not statistically different by gender $(p=0.79)$ or age group $(p=0.083)$. Patients treated with oxaliplatin $(27.9 \%)$ versus those with nonoxaliplatin $(26.3 \%)$ regimens had similar failure rates overall ( $p=0.714)$. There was no difference in the failure rates of oxaliplatin versus irinotecan-based regimens. Loss of $\mathrm{CR}$ was significantly higher for delayed $(p=0.028)$ and overall CINV $(p=0.017)$ with 
Table 1 Failure to achieve complete response for acute and delayed chemotherapy-induced nausea and vomiting across the chemotherapy cycles as per emetogenic risk groups

\begin{tabular}{|c|c|c|c|c|c|}
\hline & $\begin{array}{l}\text { Overall cohort } \\
n(\%)\end{array}$ & $\begin{array}{l}\text { Minimal risk } \\
n(\%)\end{array}$ & $\begin{array}{l}\text { LEC regimen } \\
n(\%)\end{array}$ & $\begin{array}{l}\text { MEC regimen } \\
n(\%)\end{array}$ & $\begin{array}{l}\text { HEC regimen } \\
n(\%)\end{array}$ \\
\hline \multicolumn{6}{|l|}{ Nausea } \\
\hline Acute & $180(16.9)$ & $2(10.5)$ & $27(12.3)$ & $124(20.2)$ & $27(12.8)$ \\
\hline Delayed & $192(18.1)$ & $1(5.2)$ & $28(12.7)$ & $134(21.8)$ & $29(13.7)$ \\
\hline \multicolumn{6}{|l|}{ Vomiting } \\
\hline Acute & $104(9.8)$ & $0(0)$ & $13(5.9)$ & $83(13.5)$ & $8(3.8)$ \\
\hline Delayed & $101(9.5)$ & $0(0)$ & $10(4.6)$ & $78(12.7)$ & $13(6.1)$ \\
\hline \multicolumn{6}{|l|}{ Overall CINV } \\
\hline Acute & $206(19.4)$ & $2(10.5)$ & $33(15.1)$ & $143(23.3)$ & $28(13.3)$ \\
\hline Delayed & $212(19.9)$ & $1(5.2)$ & $32(14.6)$ & $147(23.9)$ & $32(15.2)$ \\
\hline Overall (acute and delayed) & $247(23.2)$ & $2(10.5)$ & 37 (16.9) & $173(28.2)$ & $35(16.6)$ \\
\hline
\end{tabular}

Table 2 Comparison of complete response rates (acute, delayed, and overall) for cycle 1 versus cycle 2 and beyond

\begin{tabular}{|l|l|l|l|}
\hline CR not achieved & Cycle $\mathbf{1}(\boldsymbol{n}=\mathbf{7 3 8 )} ; \boldsymbol{n}(\%)$ & Cycle 2 and beyond $(\boldsymbol{n}=\mathbf{3 2 4}) ; \boldsymbol{n}(\%)$ & $\boldsymbol{p}_{\text {-Value }}$ \\
\hline Overall & $176(23.8)$ & $71(21.9)$ & 0.688 \\
\hline Acute & $141(19.1)$ & $65(20.0)$ & 0.483 \\
\hline Delayed & $156(21.1)$ & $56(17.2)$ & 0.191 \\
\hline
\end{tabular}

Abbreviation: CR, complete response.

aPearson's Chi-square test.

the capecitabine-oxaliplatin (CAPOX) regimen (once in every 3 weeks $130 \mathrm{mg} / \mathrm{m}^{2}$ oxaliplatin) as against the regimens of oxaliplatin using $85 \mathrm{mg} / \mathrm{m}^{2} 2$ weekly.

The baseline demographics of patients who failed to achieve CR as compared with those who achieved CR in their first cycle of chemotherapy. The significant differences were observed for ECOG performance status and emetogenicity group of chemotherapy on univariate analysis. Logistic regression analysis was performed to ascertain the effects of age, gender, comorbidities, ECOG group, and emetogenicity risk group on the likelihood that patients failed to achieve CR. The logistic regression model was statistically significant, $\chi 2=46.5, p<0.001$ (-Table 3 ). The model explained 9.2\% (Nagelkerke's R2) of the variance in CR through the variables included and correctly classified $77.6 \%$ of cases. Patients with an ECOG performance score of 2 were 2.1 times morelikely to fail to achieveCR than patients withascore of 0 to 1 $(p=0.011)$. Patients with an ECOG performance score of 2 $(p=0.011)$ and moderate emetogenic risk group $(p=0.006)$ significantly predicted the failure to achieve CR.

\section{Discussion}

This study was performed to assess the rates of complete control of CINV in $738 \mathrm{GI}$ cancer patients who received 1,062 cycles of chemotherapy on an outpatient basis in daycare of our hospital. A previous study from our institution reported that the proportion of prescriptions, classified as ASCO guideline adherent, and postcorrective measures was 63.6 and 98.5\%, respectively. ${ }^{8}$ In another study from Japan, Fujii et al reported a significant improvement in complete response from nausea and vomiting during the delayed period from 54 to $74 \%$ when the adherence to standard CINV prophylaxis guidelines was ensured. ${ }^{9}$ However, in our study, only patients who received CINV prophylaxis as per the standard guidelines were included. Despite adequate prophylaxis, CR could not be achieved in $23.2 \%$ of all the chemotherapy cycles. This is higher in comparison to Japanese study by Suzuki et al where they reported CR rates of $96 \%$ for no vomiting and $87 \%$ for no CINV during the overall period of the first cycle of chemotherapy.$^{10}$ This study included only colorectal cancer patients receiving MEC; however, in our study, multi-site GI cancer patients were included. Interestingly, the rates of failure to achieve CR were the highest among patients receiving MEC regimen (28.2\%) and this was statistically worse as compared with patients on $\operatorname{HEC}(16.6 \%, p=0.001)$. In the entire cohort, the comparison of failure rates to achieve CR clearly pointed toward higher rates in oxaliplatin-based chemotherapy versus nonoxaliplatin chemotherapy in acute $(p=0.042)$, delayed $(p=0.034)$, and overall $(p=0.006)$ phases of CINV. Nishimura et al tried to evaluate the role of NK1 antagonists in patients receiving oxaliplatin and reported significant benefit of the same. ${ }^{11,12}$ Another phase-III study added that NK1 antagonist casopitant intravenous single dose to ondansetron and dexamethasone in MEC regimens failed to show an improvement in CINV rates. ${ }^{4}$ Our study reinforces the fact that patients receiving oxaliplatin have higher failure rates and might benefit from additional antiemetic prophylaxis. In the study, presented as abstract by Binder et al, which evaluated 13,330 patients receiving oxaliplatin, the results concluded that there is a differential risk of CINV based on 
Table 3 Logistic regression analysis of factors that can affect the complete response rates for moderate emetogenic risk chemotherapy

\begin{tabular}{|c|c|c|c|}
\hline & Number $(n=601)$ & Percentage & $p$-Value \\
\hline \multicolumn{4}{|l|}{ Overall } \\
\hline Age (y) & & & \multirow[t]{3}{*}{0.177} \\
\hline$\leq 60$ & $137 / 475$ & 28.8 & \\
\hline$>60$ & $28 / 126$ & 22.2 & \\
\hline \multicolumn{3}{|l|}{ Gender } & \multirow[t]{3}{*}{0.588} \\
\hline Male & $102 / 385$ & 26.5 & \\
\hline Female & $63 / 216$ & 29.1 & \\
\hline \multicolumn{3}{|l|}{ Chemotherapy regimen } & \multirow[t]{3}{*}{0.714} \\
\hline Oxaliplatin based & $115 / 407$ & 28.2 & \\
\hline Nonplatin based & $50 / 190$ & 26.3 & \\
\hline
\end{tabular}

Note: Twelve patients receiving carboplatin were removed from this analysis of moderate emetogenic risk chemotherapy.

age and gender. ${ }^{13}$ In our study, there was no effect of gender or age on the CP rates.

An additional finding in our study was that among patients receiving MEC, CR rates of oxaliplatin versus irinotecan regimen were similar. These results are similar to that reported by lihara et al in which the control of CINV in the first cycle was similar among various MEC regimens, including carboplatin, irinotecan, and oxaliplatin. ${ }^{14}$ Thus, it appears that irinotecan- and oxaliplatin-based regimens might benefit from additional antiemetic prophylaxis (NK1 antagonist). In our analysis, oxaliplatin $130 \mathrm{mg} / \mathrm{m}^{2}$ ( 3 weekly) appears to be more emetogenic than $85 \mathrm{mg} / \mathrm{m}^{2}$ ( 2 weekly). The loss of CR was significantly higher (for delayed and overall CINV) with the CAPOX regimen $\left(130 \mathrm{mg} / \mathrm{m}^{2}\right.$ oxaliplatin) as against the regimens of oxaliplatin using $85 \mathrm{mg} / \mathrm{m}^{2}$. But this was not statistically significant when it comes to the acute phase. Hence this difference could be due to capecitabine, and this can be hypothesis-generating to consider CAPOX as multiday requiring additional antiemetic prophylaxis. The addition of NK1 antagonists or olanzapine can be considered for such regimens. ${ }^{12,15}$

\section{Limitations and Strengths}

The authors admit that there are some important limitations to this study. The present study was a single-center observational study. However, an important strength of this study was the exclusive patients of GI cancers and the inclusion of all risk groups in the study to provide real-world data.

\section{Conclusion}

Around one-fourth of patients failed to achieve complete response for CINV in GI cancers despite using prophylaxis as per standard guidelines. MEC regimen patients had the highest failure rates suggesting the need to revisit the guidelines for these patients.

\section{Informed Consent}

Consent was not applicable as data were collected from a prospectively collected database including patient diaries which are the parts of standard of care in our unit.

\section{Authors' Contributions}

All authors contributed equally in all aspects.

Funding

None declared.

Conflicts of Interest

None declared.

\section{References}

1 Cohen L, de Moor CA, Eisenberg P, Ming EE, Hu H. Chemotherapy-induced nausea and vomiting: incidence and impact on patient quality of life at community oncology settings. Support Care Cancer 2007;15(5):497-503

2 Lachaine J, Yelle L, Kaizer L, Dufour A, Hopkins S, Deuson R. Chemotherapy-induced emesis: quality of life and economic impact in the context of current practice in Canada. Support Cancer Ther 2005;2(3):181-187

3 Roila F, Molassiotis A, Herrstedt J, et al. participants of the MASCC/ESMO Consensus Conference Copenhagen 2015. 2016 MASCC and ESMO guideline update for the prevention of chemotherapy- and radiotherapy-induced nausea and vomiting and of nausea and vomiting in advanced cancer patients. Ann Oncol 2016;27(suppl 5) :v119-v133

4 Hesketh PJ, Kris MG, Basch E, et al. Antiemetics: American Society of Clinical Oncology Clinical Practice Guideline update. J Clin Oncol 2017;35(28):3240-3261

5 Vaid AK, Gupta S, Doval DC, et al. Expert consensus on effective management of chemotherapy-induced nausea and vomiting: an Indian perspective. Front Oncol 2020;10:400

6 Jain S, Engineer R, Ostwal V, et al. Addition of short course radiotherapy in newly diagnosed locally advanced rectal cancers with distant metastasis. Asia Pac J Clin Oncol 2020

7 Chaudhary NK, John RR, Boddu D, Mahasampath G, Nesadeepam N, Mathew LG. Palonosetron is a better choice compared with ondansetron for the prevention of chemotherapy-induced nausea and vomiting (CINV) in a resource-limited 
pediatric oncology center: results from a randomized control trial. J Pediatr Hematol Oncol 2019;41(4):294-297

8 Patil VM, Noronha V, Joshi A, et al. Adherence to and implementation of ASCO antiemetic guidelines in routine practice in a tertiary cancer center in India. J Oncol Pract 2017;13(6):e574-e581

9 Fujii H, lihara H, Ishihara M, Takahashi T, Yoshida K, Itoh Y. Improvement of adherence to guidelines for antiemetic medication enhances emetic control in patients with colorectal cancer receiving chemotherapy of moderate emetic risk. Anticancer Res 2013;33(12):5549-5556

10 Suzuki A, Kobayashi R, Fujii H, et al. Control of nausea and vomiting in patients with colorectal cancer receiving chemotherapy with moderate emetic risk. Anticancer Res 2016;36(12):6527-6533

11 Nishimura J, Satoh T, Fukunaga $M$, et al. Multi-center Clinical Study Group of Osaka, Colorectal Cancer Treatment Group (MCSGO). Combination antiemetic therapy with aprepitant/fosaprepitant in patients with colorectal cancer receiving oxaliplatin-based chemotherapy (SENRI trial): a multicentre, randomised, controlled phase 3 trial. Eur J Cancer 2015;51(10):1274-1282

12 Vaswani B, Bhagat S, Patil S, Barkate H. Effectiveness of a novel, fixed dose combination of netupitant and palonosetron in prevention of chemotherapy induced nausea and vomiting: a reallife study from India. World J Clin Oncol 2020;11(8):606-613

13 Binder G, Saunders WB. Chemotherapy-induced nausea and vomiting (CINV) - incidence by age and sex among patients receiving oxaliplatin. International Society For Pharmacoeconomics and Outcomes Research 2018;21 (suppl 1) -S14

14 Iihara $\mathrm{H}$, Ishihara $\mathrm{M}$, Fujii $\mathrm{H}$, et al. Comparison of the control of nausea and vomiting among several moderately emetic-risk chemotherapy regimens. J Cancer 2016;7(5):569-575

15 Babu G, Saldanha SC, Kuntegowdanahalli Chinnagiriyappa L, et al. The efficacy, safety, and cost benefit of olanzapine versus aprepitant in highly emetogenic chemotherapy: a pilot study from South India. Chemother Res Pract 2016;2016:3439707

\section{Monthly ACOS Seminars}

The Asian Cardio Oncology Society has a monthly seminar on the first Wednesday of every month from $700 \mathrm{pm}$ to $830 \mathrm{pm}$. The inaugural seminar is on $6^{\text {th }}$ January 2021.

To register, please visit www.acos.info or send email to asiancardiooncologysociety@gmail.com 